\title{
Superelastic NiTi honeycombs: fabrication and experiments
}

\author{
John A Shaw ${ }^{1}$, David S Grummon ${ }^{2}$ and John Foltz ${ }^{2}$ \\ ${ }^{1}$ Department of Aerospace Engineering, The University of Michigan, Ann Arbor, MI, USA \\ ${ }^{2}$ Department of Chemical Engineering and Materials Science, Michigan State University, \\ East Lansing, MI, USA
}

Received 15 July 2005, in final form 12 September 2005

Published 15 January 2007

Online at stacks.iop.org/SMS/16/S170

\begin{abstract}
In this paper we demonstrate a new class of superelastic NiTi honeycomb structures. We have developed a novel brazing technique that has allowed us to fabricate Nitinol-based cellular structures with relative densities near 5\%. Commercially available nickel-rich Nitinol strips were shape-set into corrugated form, stacked, and bonded at high temperature by exploiting a contact eutectic melting reaction involving pure niobium. After heat treatment to restore transformational superelastic response, prototype honeycomb structures were subjected to severe in-plane compression loading at room temperature. The specimens exhibited good specific strength, high specific stiffness, and enhanced shape recovery compared to monolithic shape memory alloys (SMAs).

Compressive strains of over $50 \%$ could be recovered upon unloading. The demonstrated architectures are simple examples of a wide variety of possible built-up topologies, enabled by the bonding method, that can be engineered for customizable net section properties, arbitrary shape, and kinematically enhanced thermomechanical shape-memory and superelastic response.
\end{abstract}

\section{Introduction}

Materials that combine ultra-low density with the desirable characteristics of metals have been an object of technical development for decades, and a variety of metals and alloys are commercially available in various cellular forms. Cellular structures made from shape-memory alloys (SMAs) are particularly intriguing for their potential to deliver shape memory and/or superelasticity in a lightweight material. While porous forms of NiTi have been made (Lagoudas et al 2001), the difficulty of joining Nitinol to itself has prevented the realization of built-up cellular honeycombs from NiTi-based SMAs.

Even when conventional strength and stability characteristics are all that is sought, metallic foams and honeycombs, with their light weight, high specific stiffness, and well-developed energy absorption characteristics, are of obvious utility (Gibson and Ashby 1997, Ashby et al 2000). In particular, Papka and Kyriakides $(1994,1998)$ presented interesting in-plane crushing experiments of hexagonal aluminum honeycombs ${ }^{3}$. These showed an initial stiff response, followed by a plateau

\footnotetext{
3 Although these topologies are more often loaded in the out-of-plane direction (i.e. perpendicular to a honeycomb panel) in structural and energy absorption applications, this study demonstrated interesting propagation instabilities that could be exploited as energy absorption mechanisms.
}

where crushing continued at nearly constant load. The plateau was associated with localized deformation of particular rows of cells. Shear-like bands propagated as the honeycomb densified, and the plateau ended as mutual contact of the cell walls caused the load to rise steeply. Of course, the aluminum honeycombs in these experiments suffered permanent deformation.

Some attempts to produce porous SMAs by hot isostatic pressing of powders (Lagoudas et al 2001, Thangaraj et al 2000, Li et al 2000) have achieved relative densities as low as $30 \%$, but the irregular pore shape in these materials causes stress concentrations that severely degrade the mechanical properties. More recently, a Nitinol-based material with a more regular, open-cell foam topology, and a relative density below $5 \%$, was reported (Shaw et al 2002, Grummon et al 2003). These materials were realized using a powder metallurgy technique and a polymeric foam precursor, and were shown to possess the martensitic transformation characteristics of SMAs. Unfortunately, embrittlement by interstitial contaminants prevented a useable superelastic response.

\section{Objectives}

Our objective has been to design and fabricate a material that combines the advantages of a metallic foam with the adaptive 

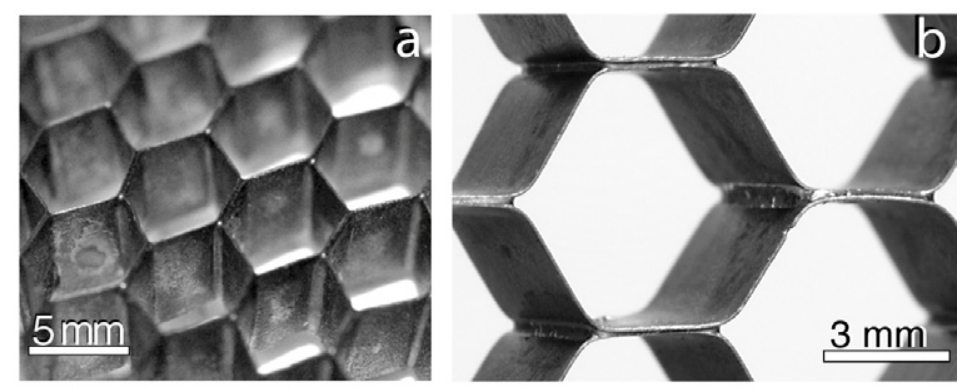

Figure 1. (a) A superelastic Nitinol honeycomb structure with relative density of $\sim 5 \%$, fabricated by joining individual corrugated Nitinol strips using the newly developed brazing system; (b) close-up image showing braze joints between adjacent lands of corrugated strips.

properties of an SMA. Besides the individual advantages of these two classes of material, the combination should provide adaptive properties beyond those of a conventional monolithic SMA. We consider that the free space inherent in cellular architectures should result in deformation kinematics that amplify thermoelastic shape recovery to well beyond the conventional monolithic strain limits, while simultaneously improving the thermal time constant by increasing the surface to volume ratio. Both attributes-isothermal recovery (superelasticity) and thermal shape recovery-are of obvious interest in the context of active structures. Furthermore, if Nitinol honeycombs could be realized as open-cell, layered structures by simply building them up from wrought SMA sheet, strip, tube, or wire (pre-engineered and acquired off-theshelf), such materials might become economically attractive. Our goal, therefore, was to fabricate shape-memory and superelastic metal honeycombs that combine high specific stiffness, high resilience and excellent fatigue resistance, with low density, good thermal and chemical stability, and biocompatibility. The discovery of a niobium-based reactive brazing technique has allowed us to fabricate prototypes of such structures, as shown in figure 1.

The maximum tensile strain recovery obtainable from a monolithic Nitinol polycrystal is in the range of $5-8 \%$, in the low-cycle limit, and is less than $2.5 \%$ when high-cycle fatigue is a factor. These limits can, however, be substantially exceeded by exploiting the bending of thin ligaments during in-plane loading of open-cell structures. Open structures also cope more effectively with latent heat effects associated with the underlying displacive transformations in SMAs. Thermal inertia tends to dominate the response time of SMA actuators, and, furthermore, can cause hypersensitive rate dependences (Shaw and Kyriakides 1995, 1997, Shaw et al 2003, Iadicola and Shaw 2004). These scale with the volume to surface area ratio, which is greatly reduced with cellular materials.

\section{A new joining method for Nitinol}

Honeycomb structures built up from wrought SMAs are a viable alternative to foamed or porous metals if a method can be found to join individual corrugated or dimpled sheets or strips. The necessary joining step to create an open topology must not only provide a robust metallurgical bond, but must also be derivable from a simple, clean, and cost-effective batch process. Given the range of potential applications, the bond should additionally have high corrosion resistance, good thermal stability, and should contain only biocompatible phases. While a few specialized techniques for soldering and welding Nitinol have been developed over the years, until now no low-cost joining method capable of producing tough metallurgical bonds in complex multiple-contact structures, such as honeycombs or spaceframes, has been available.

We have recently discovered a solution in the form of a niobium-based braze that exploits contact melting in a quasi-binary eutectic system with promising metallurgical characteristics. Essentially, we have found that when niobium is brought into contact with conventional wrought Nitinol at elevated temperature, interdiffusion between NiTi and pure $\mathrm{Nb}$ quickly leads to the formation of a liquid phase that aggressively wets both the pure niobium and NiTi, and is 'selffluxing' in the sense that it appears to dissolve oxide scales. The liquid phase flows readily into capillary fissures, and subsequently solidifies into a braze joint having good strength and ductility. The reactive braze is based on the physical metallurgy of the $\mathrm{Ni}-\mathrm{Ti}-\mathrm{Nb}$ ternary system, and specifically on the apparent existence of a quasi-binary eutectic isopleth (Prema et al 1995) involving the reaction

$$
\text { (B2) } \mathrm{NiTi}+\left(\text { bcc) } \mathrm{Nb} \rightarrow \mathrm{Ni}_{36} \mathrm{Ti}_{38} \mathrm{Nb}_{24}\right. \text { (Liq.). }
$$

Interfacial reactions of the sort in equation (1), those that form eutectic liquids at dissimilar alloy junctions, are not uncommon. However, solidification of most non-dilute ternary alloys yields complex microstructures containing embrittling intermetallic phases ${ }^{4}$. In the present process, on the other hand, the terminal phases that result from eutectic freezing of $\mathrm{Ni}_{36} \mathrm{Ti}_{38} \mathrm{Nb}_{24}$ are phases based on austenitic Nitinol and bcc niobium. Each is a familiar and well-understood metal with attractive physical and mechanical characteristics. For example, both the two-phase eutectic solid and the individual phases in this constituent are ductile and tough. Both phases are corrosion resistant (Cherghescu and Constantin 1998, Dong et al 2000) and thermally stable. None of the alloy constituents is exotic or unreasonably expensive. We will present results on the physical metallurgy of the $\mathrm{Ti}-\mathrm{Ni}-\mathrm{Nb}$ system elsewhere, and the mechanical properties of the braze material (in which rather few failures have yet been observed) are the subject of ongoing work.

4 We note that the special transformational characteristics of the B2 superlattice in $\mathrm{NiTi}$ exist in commercial alloys like $\mathrm{Ni}_{47} \mathrm{Ti}_{44} \mathrm{Nb}_{9}$, in which the effect of niobium is to beneficially widen the temperature hysteresis for SMA connectors (Melton et al 1989, Zhang et al 1990). 

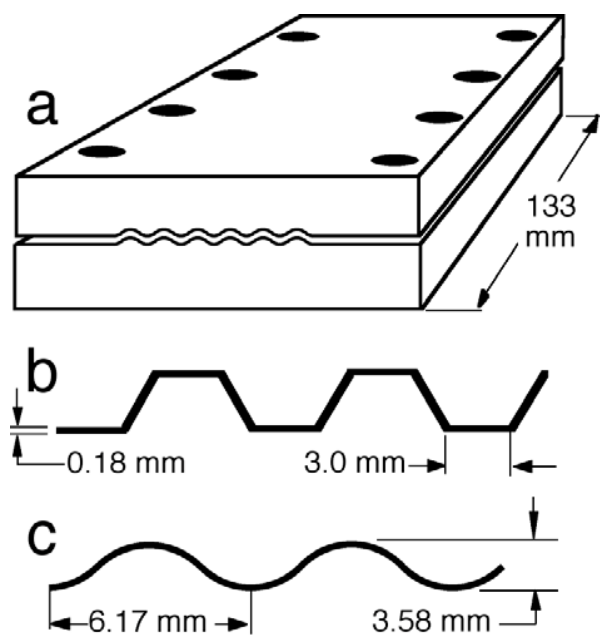

Figure 2. (a) Example of a small shape-setting die (bi-cylindrical corrugation) accommodating about twenty $75 \mathrm{~mm}$ long strips; (b) and (c) dimensions of the hexagonal and bi-cylindrical corrugation.

\section{Areas of application}

It is clear that in addition to allowing straightforward fabrication of cellular structures like that shown in figure 1, the new joining method can be adapted to form a wide variety of possible geometrical and topological forms. Complex space-frames, honeycomb sandwich panels, and other sparse built-up structures made from Nitinol, and related shapememory and superelastic alloys, should be feasible. Such materials could be designed to display either thermally driven adaptive characteristics, in shape-memory mode, and/or the isothermal constitutive response of superelastic alloys, but with kinematically amplified strain recovery. The literature indicates that the braze material is biocompatible and has good osteoconductivity (Eisenbarth et al 2004), potentially enabling a variety of compliant pseudoelastic biomedical implant and bone-replacement materials unobtainable by any other means.

SMA honeycombs may also prove useful in defense and aerospace applications as thermally active multifunctional materials, highly resilient structures, lightweight armor, or in novel vibration damping systems. The brazing method itself should be adaptable to the joining of heavy sections for civil engineering applications such as earthquake damage control. In addition, preliminary investigation indicates that the technique may be amenable to the joining of Nitinol to certain dissimilar metals such as titanium and stainless steel.

\section{Specimen fabrication}

Honeycomb specimens were fabricated at Michigan State University from $5.2 \mathrm{~mm}$ wide $\times 0.2 \mathrm{~mm}$ thick rolled nickel-rich Nitinol strip obtained from Memry Corporation, Brookfield, $\mathrm{CT}$. Based on differential scanning calorimetry the strip material had the transformation temperatures $A_{\mathrm{s}}=-25^{\circ} \mathrm{C}, A_{\mathrm{f}}=$ $-7{ }^{\circ} \mathrm{C}, M_{\mathrm{s}}=-54^{\circ} \mathrm{C}$, and $M_{\mathrm{f}}=-75^{\circ} \mathrm{C}$. Therefore, even after significant thermal processing the Nitinol material was expected to be superelastic at room temperature. The strips
Table 1. Geometric and process parameters for specimens used in the experiments.

\begin{tabular}{llll}
\hline Experiment & $\mathbf{1}$ & $\mathbf{2}$ & $\mathbf{3}$ \\
\hline Specimen ID & $030905 \mathrm{c}$ & $030905 \mathrm{~d}$ & $030805 \mathrm{a}$ \\
\hline$t(\mathrm{~mm})$ & 0.19 & 0.10 & 0.10 \\
$d(\mathrm{~mm})$ & 5.55 & 5.38 & $4.8^{\mathrm{a}}$ \\
Braze temperature $\left({ }^{\circ} \mathrm{C}\right)$ & 1175 & 1175 & 1200 \\
Braze time $(\mathrm{s})$ & 90 & 300 & $<10$ \\
Aging temperature $\left({ }^{\circ} \mathrm{C}\right)$ & 514 & 514 & 520 \\
Age time $(\mathrm{s})$ & 600 & 600 & 600 \\
\hline
\end{tabular}

a Cell size is based on the diameter of the resulting

bi-cylindrical arc.

were first shape-set into corrugated forms at $500^{\circ} \mathrm{C}$ for 10 30 min using stainless steel dies as indicated in figure 2 , which also shows details of both bi-cylindrical and hexagonal corrugation forms ${ }^{5}$ used in the present experiments. After shapesetting, the corrugated strips were etched in a solution contain-

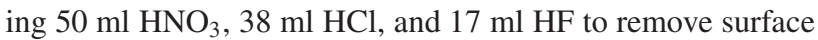
oxides $^{6}$. In the case of the hexagonally corrugated strips, the honeycomb layup was assembled with small $50.8 \mu \mathrm{m}$ thick square pieces $(\sim 3 \mathrm{~mm} \times 3 \mathrm{~mm})$ of $99.7 \%$ pure niobium foil placed between the contacting faces. Bi-cylindrically corrugated strips were assembled together with $0.127 \mathrm{~mm}$ diameter pure niobium wires on either side of each contact zone.

The assembled layup was lightly secured in TZmolybdenum support fixtures and washed in acetone and ethanol. No fluxing agents were used. Brazing was conducted in a Centorr M60 tungsten-element diffusion-pumped vacuum furnace evacuated to a base pressure of $7 \times 10^{-6}$ Torr. Once the base pressure was attained, the specimens were heated quickly to between 1175 and $1200^{\circ} \mathrm{C}$, held for a brief period, and then furnace cooled. After removal from the vacuum furnace, the as-brazed honeycombs were given an aging treatment at between 450 and $515^{\circ} \mathrm{C}$ prior to compression testing. Table 1 gives some geometric data and the processing history for each specimen tested. Note that $t$ is the nominal ligament thickness and $d$ is the nominal cell diameter. The temperatures listed should be considered approximate, since the exact temperature history of the specimen was affected by the thermal inertia of the chamber and fixtures. We do not believe, however, that the specimen temperature lagged significantly behind the chamber temperature based on the known $1170{ }^{\circ} \mathrm{C}$ temperature for the $\mathrm{NiTi}+\mathrm{Nb}$ reaction to occur. Detailed characterization of the mechanical properties of the braze and optimization of processing parameters is the subject of ongoing research and will be published elsewhere.

\section{Mechanical experiments}

Three lateral crushing experiments are presented on different specimens that were initially austenitic, one on a short hexagonal honeycomb (experiment 1), one on a taller

\footnotetext{
5 The die dimensions in this figure differ slightly from those of the final honeycombs due to spring-back and process-induced deformations.

6 Although it has been observed that bonding takes place in the presence of surface oxides (and is relatively insensitive to oxidation during vacuum processing at high temperature), removal of gross oxide scales reduces contamination of the braze material itself and probably improves braze toughness.
} 


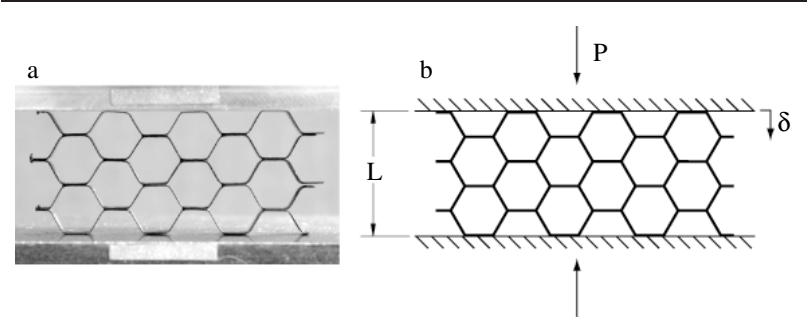

Figure 3. Experimental arrangement: (a) initial specimen image and (b) schematic of honeycomb compression experiments.

hexagonal honeycomb (experiment 2), and one on a bicylindrically corrugated honeycomb structure (experiment 3 ). The experiments were in-plane crushing tests performed at the University of Michigan using an Instron 5585 electromechanical testing machine. All experiments were performed in room temperature air $\left(22.2-22.8^{\circ} \mathrm{C}\right)$ under quasistatic displacement control. Digital optical photographs were taken periodically using a Nikon D100 camera fitted with a $105 \mathrm{~mm}$ Micro Nikkor lens.

Figure 3 shows an image of Specimen 030905c at the start of experiment 1, together with a schematic of the arrangement defining the vertical length $(L)$ between platens and the downward displacement $(\delta)$ of the upper platen. The silver strips seen on the front of the platens are reflective tags used with an EIR model LE-05 laser extensometer to accurately measure platen displacement and to serve as a crosscheck on the strain measurement derived from the crosshead displacement. Experiments 1 and 2 were performed at an average strain rate, $\dot{\delta} / L=3.33 \times 10^{-3} \mathrm{~s}^{-1}$, while experiment 3 was performed at $\delta / L=1.67 \times 10^{-3} \mathrm{~s}^{-1}$. The mechanical response is reported as the compressive load, $P$, divided

by the projected cross-sectional area, $A_{0}$ (the total footprint area, including the area of the incomplete cells at the edges), perpendicular to the loading direction, to give a macroscopic measure of compressive stress. Various strain histories were imposed to probe the effective superelastic strain recovery properties, residual strain, and cyclic ratcheting.

The results of experiment 1 are shown in figure 4 . Figure 4(a) shows the prescribed compressive strain history in which the $2 \frac{1}{2} \times 6$ cell specimen was crushed to $70 \%$ strain, unloaded to zero load, and reloaded for five cycles. The mechanical response is shown in figure 4(b) as a compression stress-strain plot. The first loading segment exhibits an initial linear response up to about $0.4 \mathrm{MPa}$, with a tangent modulus of about 7.1 MPa associated with nearly uniform deformation of the honeycomb cells. Subsequently, a knee occurs in the response and the tangent modulus decreases as the lower row of cells buckles due to stress-induced martensite transformation $(\mathrm{A} \rightarrow \mathrm{M})$. (The circled numbers in the figure correspond to times at which high-resolution digital images, shown in figure 4(c), were acquired.) As crushing proceeds the stress continues to rise between times (1a) and (1d). An angled band of deformed cells starts to form, although the specimen has too few cells to exhibit well-developed shear bands like those seen in Papka and Kyriakides (1994, 1998). Irregularities in the mechanical response correspond to events associated with buckling of certain cell rows. Note, for example, the small inward dimples that occur in the horizontal flats of the lowest cell walls. These can be seen more clearly in figure 4(d) (image (1e) at 70\% strain). Between times (1d) and (10), internal contact has occurred between some cells, causing the load to rise steeply.

At time (10) the upper platen motion is reversed to unload the specimen. The unloading tangent modulus is initially
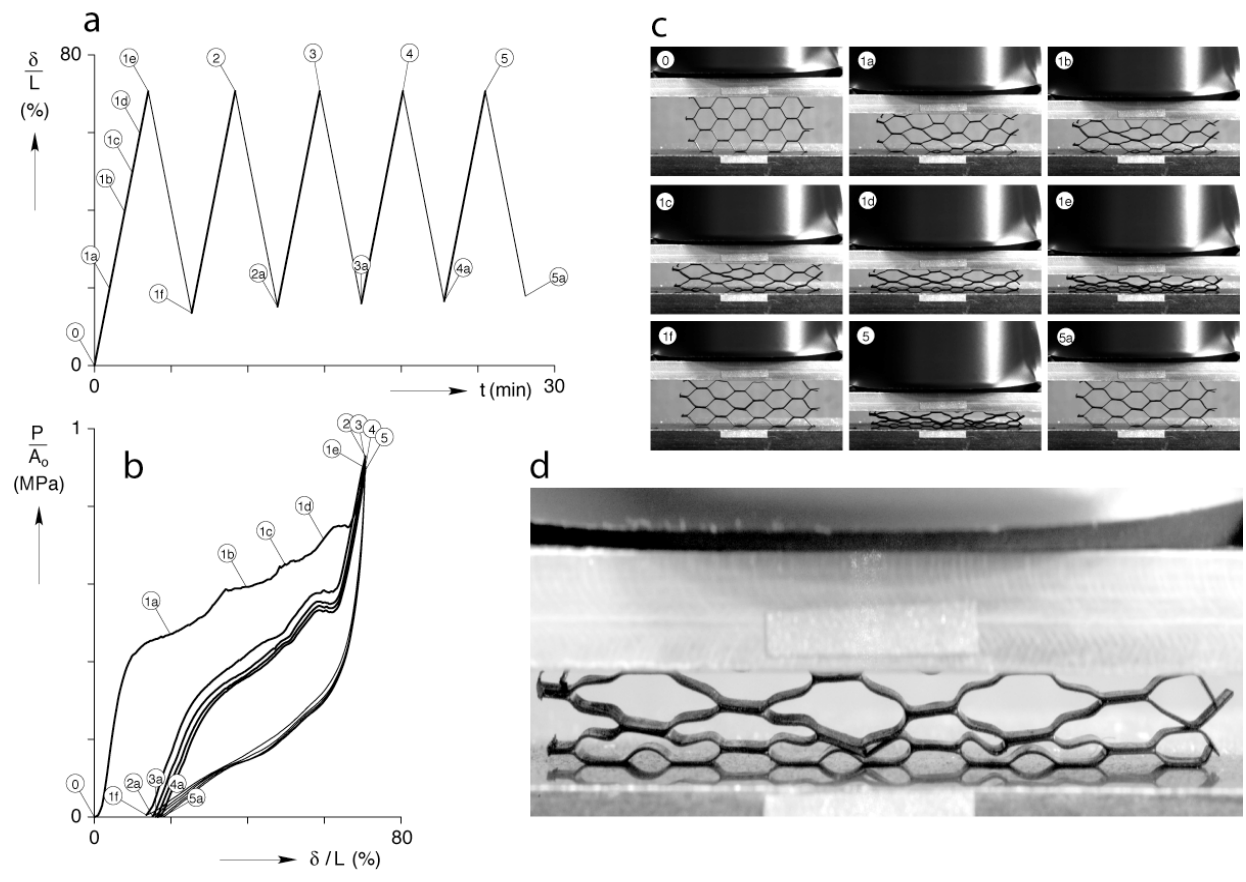

Figure 4. Results from experiment 1: (a) prescribed compressive strain history; (b) compressive mechanical response, showing shakedown of enhanced superelastic behavior with over 50\% strain recovery; (c): selected hexagonal honeycomb specimen images. Numbers correspond to states shown in figures 1(a) and (b); (d) close-up image of specimen at 70\% compressive strain (state (1) of figure 1(c)). 

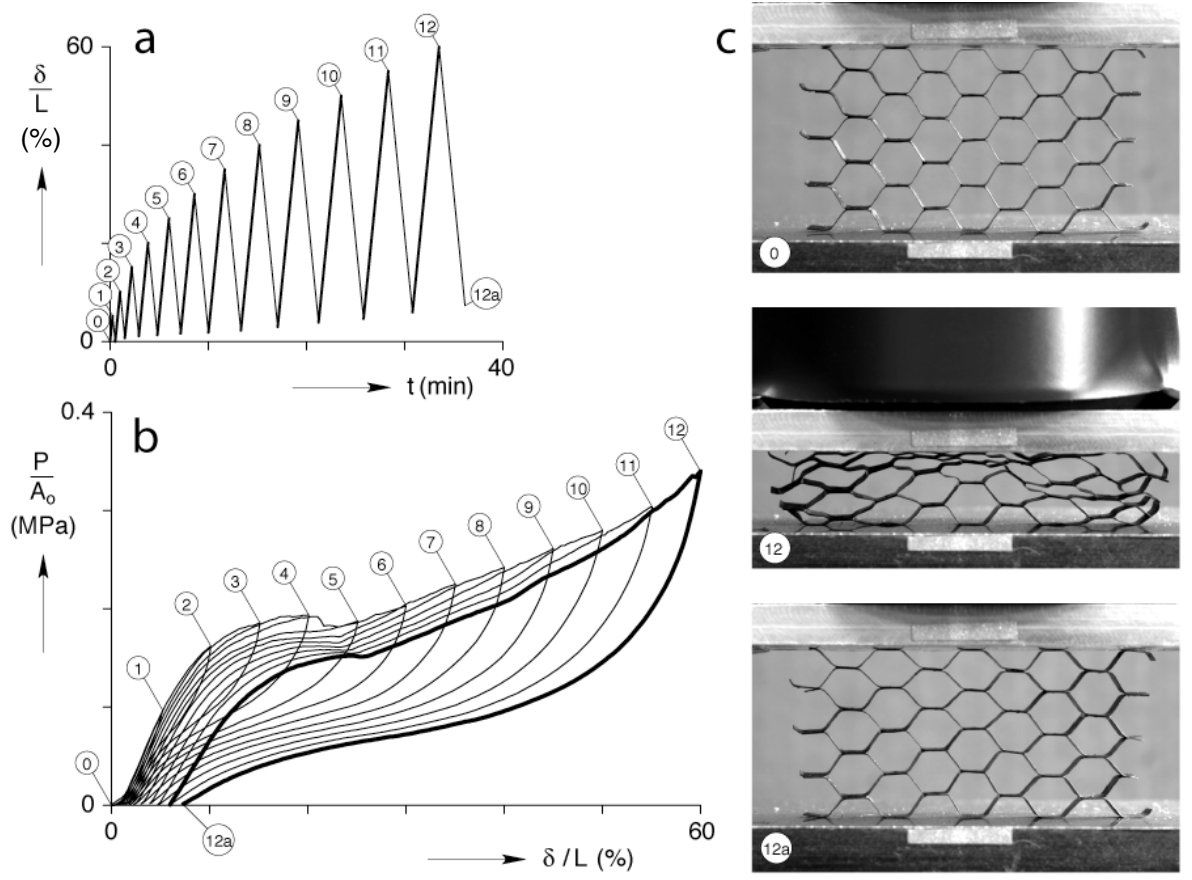

Figure 5. Results of experiment 2: (a) compressive strain history, (b) mechanical response to incremental strain cycles, (c) selected specimen images.

relatively steep (19.1 MPa), but then decreases as the reverse martensite-to-austenite $(\mathrm{M} \rightarrow \mathrm{A})$ transformation occurs. After complete unloading the residual strain is $13.6 \%$. This first cycle shows a wide hysteresis, but in subsequent loadunload cycles the hysteresis diminishes sharply, and nearly repeatable mechanical response is obtained. A small amount of transformational softening and cyclic ratcheting still occurs, however, and the residual strain is $\sim 17.9 \%$ after the fifth cycle (point (5a)). The lack of perfect cyclic stability is of some concern for eventual application, yet it is possible to perform thermomechanical processing to shake out this behavior (as will be shown in experiment 3). Cyclic stability can also be improved further by developing optimal heat treatments. Nevertheless, the repeated recovery of over 50\% strain upon unloading clearly demonstrates, for the first time, a superelastic Nitinol honeycomb.

The results of experiment 2 are shown in figure 5, which includes the imposed strain history, mechanical response, and selected specimen images during crushing and recovery. In this case a similar hexagonal honeycomb specimen was used, except that the brazing time during manufacture was longer than for the previous specimen (300 s compared to $90 \mathrm{~s}$ at $\left.1175^{\circ} \mathrm{C}\right)$, the ligament thickness was thinner $(t=0.1 \mathrm{~mm}$ compared to $0.19 \mathrm{~mm})$, and the specimen had more cells $\left(4 \frac{1}{2}\right.$ cells high by about 7 cells wide). The deformation history is comprised of incremental strain cycles, in 5\% steps, with residual strain accumulation measured after each unloading cycle.

Stress-strain response is shown in figure 5(b). A limit load is seen in the response on the fifth cycle, near $22 \%$ strain, where a local band of highly deformed cells has formed, yet the honeycomb recovers to a uniform configuration upon unloading. The final cycle, taken to $60 \%$ compressive strain, is shown by the bold line in figure 5(b). Although the closure of the loop is not perfect (leaving about $1.3 \%$ unrecovered strain), it clearly shows a wide hysteresis and nonlinear behavior typical of a superelastic material undergoing bending deformation.

Figure 5(c) shows a series of optical images of the specimen, at (0) before loading, at (12) for $60 \%$ strain, and at 12a corresponding to the final unloaded configuration with about $7.3 \%$ residual strain. Interestingly, the deformed configuration (12 displays a generally symmetric pattern with respect to a vertical plane, with a single relatively undeformed cell at the lower middle location.

The results of experiment 3 are shown in figures 6 and 7 . The specimen was a $4 \frac{1}{2} \times 11$ cell bi-cylindrically corrugated honeycomb. The brazing of this specimen was performed at $1200^{\circ} \mathrm{C}$ for just $1 \mathrm{~s}$, but the specimen had a similar aging treatment as the previous two. The ligament thickness was about $0.1 \mathrm{~mm}$, similar to the specimen used in experiment 2 . The experiment was conducted in stages. Figure 6(a) shows results from four initial cycles in which the first three are incremental in 5\% strain steps and the fourth is a repeat cycle at $15 \%$ strain. The residual strain is less than $0.2 \%$. Figure $6(\mathrm{~b})$ shows subsequent incremental cycle numbers 5 to 11 using larger scales on both the strain and stress axes. The residual strain after cycle 11 is about $8.2 \%$. Figure 6(c) shows cycles 12 through 15 taken repeatedly to a maximum strain of $50 \%$, and the residual strain after cycle 15 is about $11.2 \%$. By cycle 15 the mechanical response has nearly stabilized to a limit cycle as the rate of change of residual strain has slowed. Figure 6(d) shows three subsequent incremental cycles (16 through 18) followed by repeated cycling to $70 \%$ strain (cycles 19 through 22). Figure 6(e) shows selected images acquired during the experiment: the initial unloaded specimen at (0), the specimen 
Superelastic NiTi honeycombs
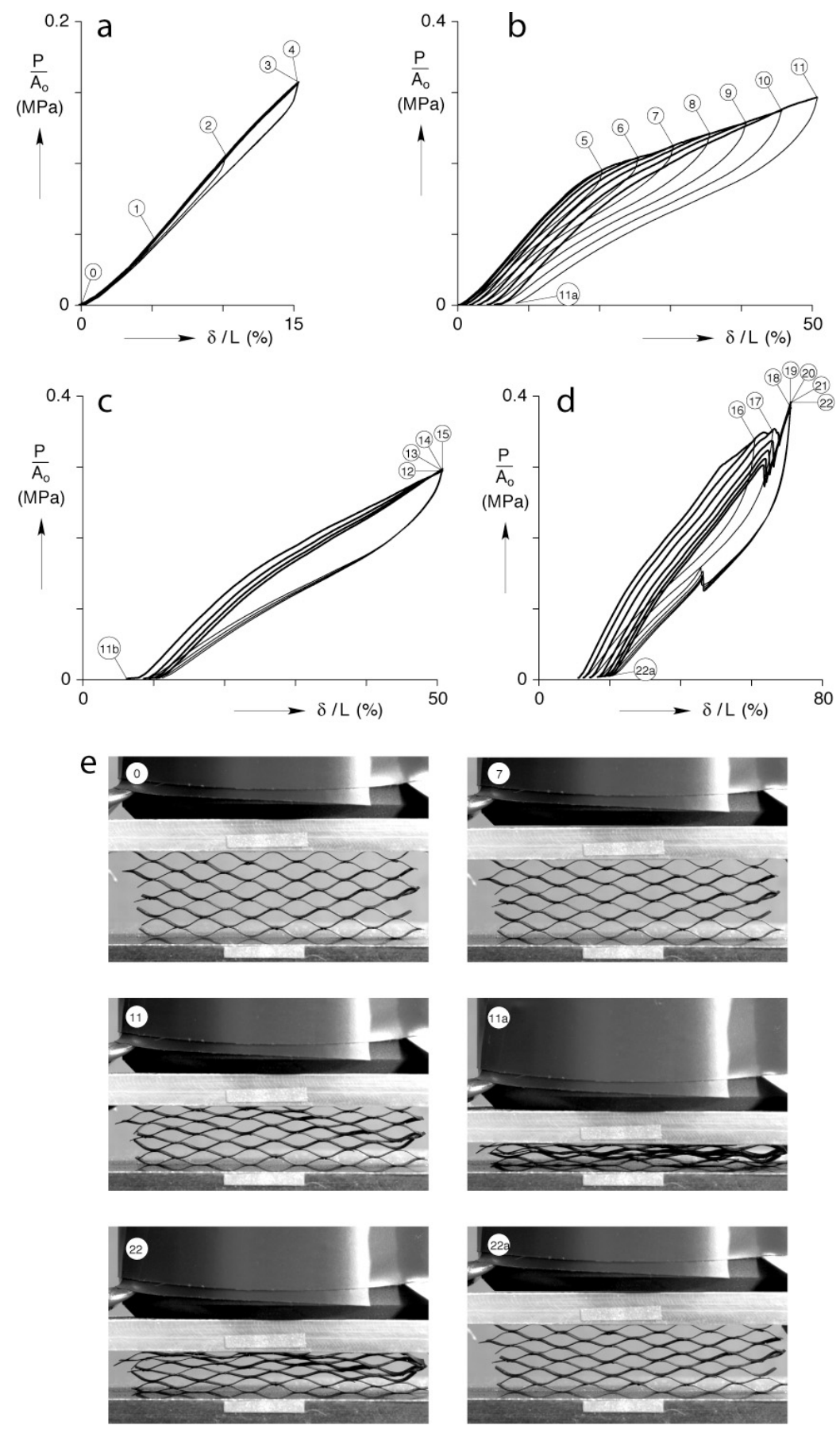

Figure 6. Results of experiment 3: (a)-(d) mechanical response of bi-cylindrical corrugated specimen: (a) three incremental cycles and then one repeated cycle to $15 \%$ compressive strain, (b) subsequent incremental cycles 5 through 11, (c) cycles 12 through 15 repeated to $50 \%$ strain, showing shakedown behavior and slight, but slowing, strain ratcheting, (d) incremental cycles 16 through 18 and then repeated cycles 19 to 22 to $70 \%$ compressive strain. Residual strain is near 20\% (note changes in scales between plots); (e) selected images of specimen.

crushed to $30 \%$ at 7 and $50 \%$ at 11 (note the formation of a band of highly deformed cells in the image), the unloaded specimen after cycle 11 at (11a), the specimen crushed to $70 \%$ at 22 , and finally the unloaded specimen at 22a after 22 cycles showing $\sim 20.2 \%$ residual strain.

As seen in figure 6(d) the stress-strain response abruptly steepens as the compressive strain nears $70 \%$, when significant internal contact between layers begins to occur (see again image 22 in figure 6(e)). This strain may be compared to the theoretical strain at full densification, which is about $93 \%$ based on the ligament thickness. The sudden drop in stress near $65 \%$ strain is apparently an unloading event associated with the change in deformation from a heterogeneous pattern to a more homogeneous one corresponding to the exhaustion of a shear band. Then the response stiffens due to significant internal contact. Similarly, the sudden rise in stress during 

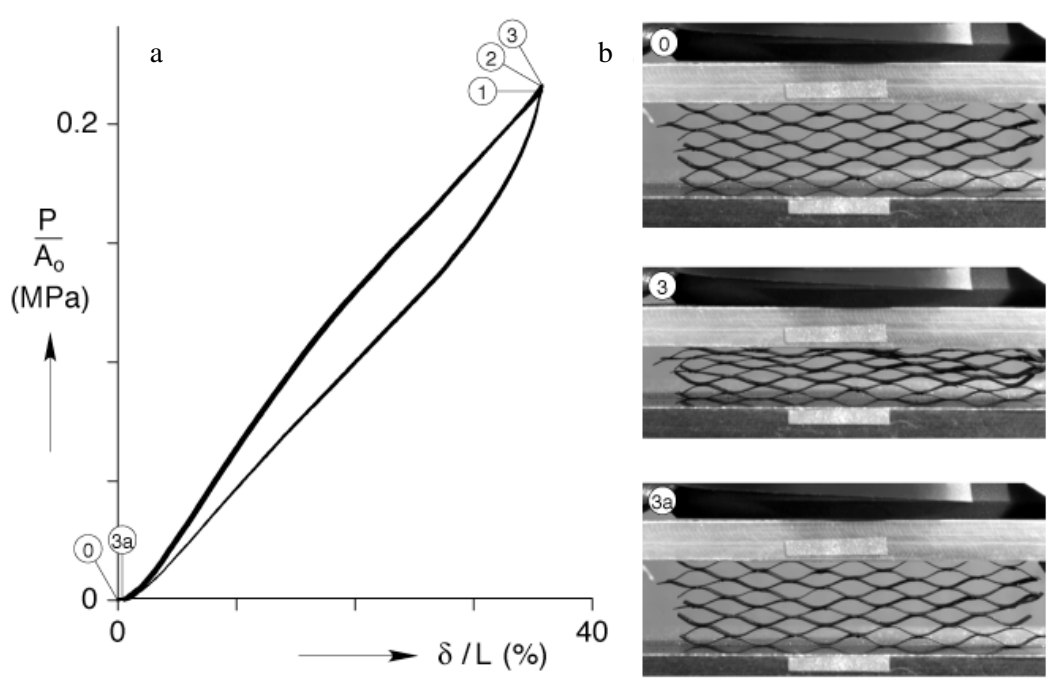

Figure 7. (a) Subsequent compressive response of specimen used in experiment 3 after renormalizing the strain by the new gage length. Three cycles show excellent repeatability; (b) selected images of specimen corresponding to numbers in figure 4(a).

Table 2. Summary showing initial tangent modulus $\left(E_{\mathrm{t}}^{*}\right)$, effective elastic modulus $\left(E^{*}\right)$, effective stress at onset of transformation $\left(\sigma^{*}\right)$, geometric factors $(t / d)^{3}$ and $(t / d)^{2}$, and corresponding scaling factors for modulus and onset. Note that properties are normalized by $E_{\mathrm{A}}=70 \mathrm{GPa}$ and $\sigma_{0}=400 \mathrm{MPa}$.

\begin{tabular}{|c|c|c|c|c|c|c|c|}
\hline \multirow[b]{2}{*}{ Experiment } & \multirow{2}{*}{$\begin{array}{l}E_{\mathrm{t}}^{*} \\
\text { (load) } \\
\text { (MPa) }\end{array}$} & \multirow{2}{*}{$\begin{array}{l}E^{*} \\
\text { (unload) } \\
\text { (MPa) }\end{array}$} & \multirow[b]{2}{*}{$\begin{array}{l}\sigma^{*} \\
(\mathrm{MPa})\end{array}$} & \multicolumn{2}{|c|}{ Geometric factors } & \multicolumn{2}{|c|}{ Scale factors } \\
\hline & & & & $\begin{array}{l}(t / d)^{3} \\
\left(\times 10^{-6}\right)\end{array}$ & $\begin{array}{l}(t / d)^{2} \\
\left(\times 10^{-4}\right)\end{array}$ & $\frac{E^{*} / E_{\mathrm{A}}}{(t / d)^{3}}$ & $\frac{\sigma^{*} / \sigma_{0}}{(t / d)^{2}}$ \\
\hline 1 & 7.1 & 19.1 & 0.35 & 41 & 12 & 6.6 & 0.73 \\
\hline 2 & 2.2 & 3.60 & 0.10 & 6.7 & 3.6 & 7.6 & 0.69 \\
\hline 3 & 1.2 & 3.64 & 0.13 & 9.0 & 4.3 & 5.8 & 0.77 \\
\hline $\begin{array}{l}3 \mathrm{a} \\
\text { (after heating) }\end{array}$ & 0.8 & 2.2 & 0.10 & 9.0 & 4.3 & 3.4 & 0.58 \\
\hline
\end{tabular}

unloading near $46 \%$ strain appears to be a deformation mode shift from homogeneous to heterogeneous (at the onset of a deformation band) as the reverse transformation $(\mathrm{M} \rightarrow \mathrm{A})$ unloads cell rows one at a time. Interestingly, similar features have been observed in tensile experiments on NiTi wires where transformation exhibits propagating fronts that coalesce on loading and nucleate during unloading (see, for example, Shaw and Kyriakides (1997)).

Following the experiment just described, removing the specimen and heating in air to about $350^{\circ} \mathrm{C}$ for $15 \mathrm{~s}$ caused a thermal strain recovery of $5.6 \%$ via the shape-memory effect. This reduced the final unrecoverable strain to $14.6 \%$, indicating that some martensite remained in the specimen following the compression cycles. This clearly demonstrates that the deformation of the honeycomb involved stress-induced martensite.

At this point the gage length was redefined to $L=$ $10.4 \mathrm{~mm}$ to reflect the new set shape, as if the previous thermomechanical history were a preconditioning regimen. Three subsequent mechanical cycles were then performed to $35.8 \%$ strain as shown in figure 7(a), with three selected images of the specimen shown in figure 7(b). The repeatability of the cyclic response is seen to be excellent, producing essentially no residual strain. The response has a smaller hysteresis than the virgin material. Once preconditioned in this way, the shape of the stress-strain curve resembles that of a filled elastomer.

A summary of selected effective properties is presented in table 2, including the initial tangent modulus $\left(E_{\mathrm{t}}^{*}\right)$, effective elastic modulus $\left(E^{*}\right)$ upon initial unloading, and effective stress at onset of transformation $\left(\sigma^{*}\right)$. In all experiments, the tangent modulus on initial loading is lower than the unloading modulus, especially in the first cycle, due to the hysteretic behavior of the material and nonlinearity arising from the changing contact area with the platens. The unloading modulus is expected to provide a better indicator of the true elastic behavior of the material.

According to simple beam-bending arguments (Gibson and Ashby 1997) the effective elastic modulus $\left(E^{*}\right)$, for a given material and honeycomb cell geometry, scales according to

$$
\frac{E^{*}}{E_{\mathrm{A}}} \propto\left(\frac{t}{d}\right)^{3},
$$

where $E_{\mathrm{A}}(=70 \mathrm{GPa})$ is the modulus of the monolithic material (austenite), $t$ is the ligament thickness, and $d$ is the honeycomb cell size. However, the stress at the onset of transformation 
$\left(\sigma^{*}\right)$ is expected to scale as

$$
\frac{\sigma^{*}}{\sigma_{0}} \propto\left(\frac{t}{d}\right)^{2}
$$

where $\sigma_{0}(=400 \mathrm{MPa})$ is taken as a characteristic transformation stress. One can see that the scale factors for the modulus and transformation-onset stress roughly agree for the first three experiments, differing by about $25 \%$ and $10 \%$, respectively. The values for experiment $3 \mathrm{a}$ (after full thermal recovery) are lower, as expected, due to the previous thermomechanical history of the specimen.

The size of the specimens that could be produced for our study was limited by the available materials, the size of fabrication fixtures, and the size of the hot zone in the vacuum chamber. Consequently, the numbers of cells in all the specimens shown here are probably too few to truly represent the bulk response, i.e., a honeycomb of infinite extent. The measured responses of our specimens were certainly affected by the irregular lateral edges, where the cells and bonding were incomplete, and by some friction with the platens at the bottom and top ends of the specimen. The former effect reduced the measured force response compared to the bulk behavior, while the latter effect increased the measured force. We suspect that the 'soft' lateral edge was the larger effect, thereby resulting in an underestimate of the force and stiffness compared to the bulk response. If possible, future experiments should be performed on specimens with more cells in both in-plane directions to reasonably measure the bulk response. Nevertheless, the focus of the current study was to demonstrate an amplified form of superelasticity in Nitinol honeycombs, which is still convincingly shown by the current specimens.

It is natural to compare the response we have observed for superelastically deforming honeycombs to what ought to be possible using conventional metals with high strength and stiffness. Consider a honeycomb structure with the same cell size and topology made from high strength steel with a modulus $E=200 \mathrm{GPa}$ and yield strength of $1 \mathrm{GPa}$. In this case, yielding can be avoided by designing the honeycomb such that the maximum elastic strain does not exceed $\sim 0.5 \%$ during crushing. Large-deformation compression of the honeycomb relies primarily on bending of the ligaments as the corrugation rows are flattened. The extreme fiber strain is proportional to $t / d$, since $d$ is a measure of the radius of curvature of the initial cell. Because the steel must remain within $0.5 \%$ strain, but the SMA may be strained to $\sim 5 \%$, the ligament thickness in the steel honeycomb must be thinner than the corresponding SMA thickness by a factor of 10 . Thus, the thinner ligament in the steel honeycomb reduces its effective modulus by a factor of $(10)^{3}=1000$, according to equation (2). Although steel has an elastic modulus greater than NiTi by a factor of $\sim 3$, the stiffness of the steel honeycomb would be less than that of the SMA structure by a factor of $1000 / 3=330$.

\section{Conclusions}

It is shown for the first time that built-up honeycomb structures with $\sim 5 \%$ relative density can be fabricated from conventional wrought Nitinol material. Long-standing difficulties with the joining of Nitinol to itself were resolved by using a newly discovered niobium-based brazing technique. The structures, after post-braze heat treatment, were shown to possess amplified transformational superelasticity when subject to severe in-plane compression. Superelastically recoverable bending strain in the Nitinol ligaments spanning between brazed joints allowed isothermal recovery of compressive strains that were a factor of ten greater than can be extracted from monolithic Nitinol. The findings have broad potential significance for lightweight armor, general energy absorption materials, high-displacement actuators, smart/active structures, and for infusable, compliance-matched biomedical implants.

\section{Acknowledgments}

J Shaw acknowledges the financial support of the Office of Naval Research (ONR young investigator grant, N00014-01-10581) and the National Science Foundation (CAREER grant, CMS-9984189) with sincere thanks.

\section{References}

Ashby M F, Evans A, Fleck N A, Gibson L J, Hutchinson J W and Wadley H N G 2000 Metal Foams: A Design Guide (London: Butterworth-Heinemann)

Cherghescu I and Constantin V 1998 Corrosion behavior of a shape memory alloy of the form $\mathrm{Ni}_{50} \mathrm{Ti}_{48} \mathrm{Nb}_{2}$ in various chemical media UPB Sci. Bull. B 60 137-41

Dong Z, Liu W, Jia D, Tang Z and Wang D 2000 Corrosion resistance of the $\mathrm{Ni}-\mathrm{Ti}-\mathrm{Nb}$ based shape memory alloys Rare Metal Mater. Eng. 29 182-4

Eisenbarth E, Velten D, Muller M, Thull R and Breme J 2004 Biocompatibility of beta-stabilizing elements of titanium alloys Biomaterials 25 5705-13

Gibson L G and Ashby M F 1997 Cellular Solids: Structure and Properties 2nd edn (Cambridge: Cambridge University Press)

Grummon D S, Shaw J A and Gremillet A 2003 Low-density open-cell foams in the NiTi system Appl. Phys. Lett. 82 2727-9

Iadicola M A and Shaw J A 2004 Rate and thermal sensitivities of unstable transformation behavior in a shape memory alloy Int. J. Plast. 20 577-605

Lagoudas D, Entchev P, Vandygriff E, Qidwai M and De-Giorgi V 2001 Modeling of thermomechanical response of porous shape memory alloys Proc. Adaptive Structures and Material Systems Symp., ASME Int. Mechanical Engineering Congr. and Exposition (New York: ASME)

Li B, Rong L, Li Y and Gjunter V E 2000 Synthesis of porous Ni-Ti SMA by SHS: reaction mechanism and anisotropy in pore structure Acta Mater. 48 3895-904

Melton K N, Proft J L and Duerig T W 1989 Wide hysteresis shape memory alloys based on the Ni-Ti-Nb system Shape Memory Materials, Proc. MRS Int. Mtg on Advanced Materials (Tokyo, May-June 1988) vol 9, pp 165-70

Papka S D and Kyriakides S 1994 In-plane compressive response and crushing of honeycomb J. Mech. Phys. Solids 42 1499-532

Papka S D and Kyriakides S 1998 Experiments and full-scale numerical simulations of in-plane crushing of a honeycomb Acta Mater. 46 2765-76

Prema S B, Tret'yachenko L A and Petyukk V M 1995 Investigation methods and properties of powdered materials: phase relations in the $\mathrm{Ti}-\mathrm{TiNi}-\mathrm{NbNi}-\mathrm{Nb}$ region of the ternary system Ti-Nb-Ni Powder Metall. Met. Ceram. 34155

Shaw J A, Chang B-C, Iadicola M A and Leroy Y M 2003 Thermodynamics of a 1-D shape memory alloy: modeling, experiments, and application Proc. SPIE 10th Annual Int. Symp. on Smart Structures and Materials (San Diego, CA, March 2003) (Bellingham, WA: SPIE Optical Engineering Press) 
Shaw J A, Gremillet A and Grummon D S 2002 The manufacture of NiTi foams ASME Adaptive Structures \& Materials Systems Symp., ASME Winter Mtg (New Orleans, LA, Nov. 2002) (New York: ASME)

Shaw J A and Kyriakides S 1995 Thermomechanical aspects of NiTi J. Mech. Phys. Solids 43 1243-81

Shaw J A and Kyriakides S 1997 On the nucleation and propagation of phase transformation fronts in a NiTi alloy Acta Mater. $45683-700$
Thangaraj K, Chen Y C and Salama K 2000 Fabrication of porous NiTi shape memory alloy by elemental powder sintering Proc. Adaptive Structures and Material Systems Symp., ASME Int. Mechanical Engineering Congr. and Exposition (Nov. 2000) vol 60 (New York: ASME) pp 59-63

Zhang C S, Zhao L C, Duerig T W and Wayman C M 1990 Effects of deformation on the transformation hysteresis and shape memory effect in a $\mathrm{Ni}_{47} \mathrm{Ti}_{44} \mathrm{Nb}_{9}$ alloy Scr. Metall. Mater. 24 1807-12 